\title{
Correspondence
}

\section{Diaphragmatic paralysis due to spinal muscular atrophy}

Sir,

We read with interest the paper on diaphragmatic paralysis due to spinal muscular atrophy by McWilliam et al. ${ }^{1}$ Their report of patients with diaphragmatic paralysis in association with a clinical picture suggestive of spinal muscular atrophy is of particular interest, since we have also been aware of this association over the past five years.

A girl born in 1981 was admitted to hospital at the age of 2 months with respiratory difficulty and poor feeding. At the time of presentation she was profoundly hypotonic and areflexic. A chest radiograph showed elevation of both diaphragms and there was poor diaphragmatic movement bilaterally on radiographic screening. Despite plication of the right hemidiaphragm (which was most severely affected) she gradually deteriorated and died at the age of 5 months of a staphylococcal pneumonia. Postoperatively she remained ventilator-dependent and showed progressive hypotonia and muscle wasting, typical of spinal muscular atrophy. A diaphragmatic muscle biopsy obtained at thoracotomy showed partial denervation atrophy, and at necropsy skeletal muscle biopsy showed similar changes and there were reduced numbers of anterior horn cells in the spinal cord sections.

A second infant, a boy, was admitted at the age of 8 weeks after a rapid respiratory deterioration. He had a two week history of snuffles, cough, and poor feeding. He was extremely hypotonic and areflexic, and it was noted from the family history that a sibling had died at the age of 9 weeks of an undiagnosed respiratory illness. The chest radiograph showed elevation of the right dome of the diaphragm. Efforts to wean him from ventilation including plication of the right diaphragm were unsuccessful. The child died at the age of 9 weeks with progressive peripheral muscle wasting and respiratory failure. Biopsy of the diaphragm taken at thoracotomy showed partial denervation atrophy, and examination of skeletal muscle at necropsy confirmed these findings.

In the light of our experience with these patients, post mortem muscle histology was performed on two further infants in whom a clinical diagnosis of spinal muscular atrophy had been made. In both these cases the diaphragm muscle had seemed thin and showed changes of denervation atrophy, although radiological diaphragmatic movement had been evident in life.

Mellins et $a l^{2}$ first described the association between diaphragmatic paralysis and spinal muscular atrophy. The report by McWilliam et al, ${ }^{1}$ together with our experience, would support the view that this association is not as rare as hitherto believed. The proposal that this condition should be considered in cases of sudden infant death seems appropriate. In two of our infants there was an acute onset of respiratory symptoms. Furthermore, we would emphasise that spinal muscular atrophy should be considered in all infants with eventration of the diaphragm, and that diaphragmatic muscle biopsy should be taken at the time of surgical plication. Denervation changes were present in diaphragmatic muscle biopsies in all our patients (two obtained before death), although in one infant skeletal muscle biopsy from the vasculus lateralis showed normal histology. In addition to diaphragmatic denervation atrophy, this patient showed loss of anterior horn cells in the lower thoracolumbar region on serial section of the spinal cord.

\section{References}

${ }^{1}$ McWilliam RC, Gardner-Medwin D, Doyle D, Stephenson JBP. Diaphragmatic paralysis due to spinal muscular atrophy. An unrecognised cause of respiratory failure in infancy? Arch Dis Child 1985;60:145-9.

${ }^{2}$ Mellins RB, Hays AP, Gold AP, Burdon WE, Bowdler JD Respiratory distress as the initial manifestation of WerdnigHoffman disease. Pediatrics 1974;53:33-40.

$$
\begin{array}{r}
\text { N P Murphy, D C Davidson And J Bouton } \\
\text { Alder Hey Children's Hospital, } \\
\text { Eaton Road, } \\
\text { Liverpool L12 } 2 A P
\end{array}
$$

\section{Dr McWilliam comments:}

I was most interested to hear of three further cases of this disorder which I regard as a distinct genetic variant of spinal muscular atrophy rather than an association of diaphragmatic paralysis with spinal muscular atrophy type 1 . The two cases described raise the important issues of early diagnosis and subsequent management. Our experience suggests that with appropriate electrophysiological investigations (motor nerve conduction studies and comprehensive electromyography) diagnosis is possible without the need for histological examination of the diaphragm. With awareness of the diagnosis I would suggest that neither ventilatory support nor plication of the diaphragm are appropriate. I certainly agree that this diagnosis should be considered in all cases of diaphragmatic paralysis or eventration, particularly as hypotonia, if present, may be considered secondary to the respiratory disorder and therefore dismissed.

\section{Perinatal hepatitis B virus detection by hepatitis $B$ virus-DNA analysis}

Sir,

We read with interest the paper by De Virgiliis et al comparing this new technique with hepatitis $\mathrm{Be}$ antigen 